Mots. Les langages du politique

\title{
Un aspect particulier de la planification linguistique : la sélection d'un système d'écriture
}

A peculiar aspect of linguistic planning: choosing a writing system

Un aspecto particular de la planificación lingüística : la selección de un sistema de escritura

\section{Ksenija Djordjevic}

\section{(2) OpenEdition}

\section{Journals}

Édition électronique

URL : https://journals.openedition.org/mots/4413

DOI : $10.4000 /$ mots. 4413

ISSN : 1960-6001

Éditeur

ENS Éditions

\section{Édition imprimée}

Date de publication : 1 mars 2004

Pagination : 59-71

ISBN : 2-84788-056-9

ISSN : 0243-6450

\section{Référence électronique}

Ksenija Djordjevic, « Un aspect particulier de la planification linguistique : la sélection d'un système d'écriture ", Mots. Les langages du politique [En ligne], 74 | 2004, mis en ligne le 24 avril 2008, consulté le 23 avril 2022. URL : http://journals.openedition.org/mots/4413; DOI : https://doi.org/10.4000/ mots. 4413 
Ksenija DJORDJEVIC ${ }^{1}$

\title{
Un aspect particulier de la planification linguistique: la sélection d'un système d'écriture
}

\begin{abstract}
À la suite de l'effondrement du bloc communiste en Europe centrale et orientale, et parallèlement au développement d'un nationalisme linguistique, le «nationalisme scriptural» a également fait son apparition. Ce dernier est moins virulent, peut-être moins souvent objet d'actualité ou de débat hors des aires culturelles concernées, bref, moins visible, mais ses conséquences sont tout aussi importantes. Certes, la géographie des écritures est incontestablement moins complexe que la géographie des langues. L'Europe «des quinze» n'a-t-elle pas plusieurs langues officielles et deux alphabets seulement, dont l'un, latin, largement majoritaire et l'autre, hellénique, réduit au territoire de la Grèce? L'écriture apparait, en Europe en tout cas, comme une modalité particulière du contact de langues, comme une dimension située bien au-delà des langues et de leurs corrélats nationaux. Un système d'écriture (alphabet, écriture syllabaire, idéogrammes) peut couvrir de nombreuses langues, sur une aire culturelle étendue. Il nous semble que les écritures ont une autre dimension, une dimension de rayonnement culturel, et en tant que telles, elles sont plus sensibles à la reconfiguration des blocs stratégiques.

Si l'on considère que les trois principaux pôles de cette reconfiguration sont le pôle de la cyrillisation, celui de la latinisation et celui de l'arabisation, correspondant à trois aires culturelles, nous pouvons nous donner pour objectif de les analyser sur un espace restreint, comme celui de l'espace yougoslave autrefois et aujourd'hui. Ce territoire est un véritable carrefour de plusieurs civilisations où se sont «rencontrés» les langues et les alphabets au fil de l'histoire. En effet, on peut observer, dans cet espace, une situation paradoxale: les Serbes et les Croates ne parleraient plus la même langue; la langue
\end{abstract}

1. ARSer/Laboratoire DIPRALANG, Université Paul-Valéry, Montpellier 3 (ksenia @wanadoo.fr) 
bosnienne a émergé et s'ébauche même une langue monténégrine... De plus, chacune de ces langues devrait une fois de plus se doter d'un système d'écriture propre et unique et le bialphabétisme (coexistence et pratique des deux alphabets) de la langue serbo-croate ne suscite plus l'enthousiasme chez ses locuteurs. L'histoire de chacun de ces peuples devrait recommencer en opposition avec les modèles passés. Par ailleurs, les nations participant à ce qui fut la communauté linguistique serbo-croate se lancent, après l'éclatement du pays, dans une lutte contre le symbole même de leur passé commun, la langue serbocroate qu'ils ont partagée autrefois, et à travers elle, ses deux alphabets, cyrillique et latin.

Le dernier cycle de guerres sanglantes balkaniques a montré que les alphabets étaient particulièrement la cible des nationalistes. L'alphabet latin et l'alphabet cyrillique se sont vus identifiés à l'ethnie et à la nation ou à la religion ennemies (Thomas, 1998, p. 113), mobilisés désormais dans le cadre des représentations identitaires des langues et des nationalismes, et ont été traités comme emblèmes nationaux ou confessionnels, si bien que le bialphabétisme n'est plus perçu comme double compétence de lecture et d'écriture qui contribue à la richesse culturelle d'une communauté plurielle. Notre objectif est de montrer dans un premier temps, après avoir défini les types d'enjeux que représentent les alphabets dans un aménagement linguistique, comment ils ont été utilisés à travers l'histoire et manipulés à des fins de domination politique dans l'espace yougoslave. Dans un deuxième temps, nous envisageons de vérifier le degré de validité de notre observation en comparant cette situation avec des situations similaires, ailleurs.

\section{L'aménagement linguistique du corpus}

Il est bien connu que la diversité des langues est bien plus grande que la diversité des États, qu'il n'y a pas de concordance entre les frontières étatiques et les frontières linguistiques. Pourtant, on a pris l'habitude de considérer «qu'un État se devait d'avoir, bien protégées derrière ses frontières, une seule nation, une seule langue» (Calvet, 1993, p. 10). On a pris également l'habitude de considérer qu'à la langue devait correspondre un système d'écriture dont le choix n'est pas neutre. Il est clair que derrière l'envie ou l'obligation d'écrire en cyrillique ou en alphabet latin, peuvent se cacher aussi bien les enjeux politiques et géostratégiques des États que les revendications identitaires de la population - encore faudrait-il définir de quelle tranche de population l'on parle.

Avant de pousser plus loin notre réflexion, il convient de s'arrêter un moment sur ce que recouvrent les expressions politique linguistique et planifi- 
cation linguistique. Louis-Jean Calvet distingue la notion de politique linguistique comme détermination des grands choix en matière de rapports entre les langues et la société, de la planification linguistique, qui désignerait la mise en pratique d'une telle politique.

Nous considérons la politique linguistique comme l'ensemble des choix conscients effectués dans le domaine des rapports entre langue et vie sociale, et plus particulièrement entre langue et vie nationale, et la planification linguistique comme la recherche et la mise en œuvre des moyens nécessaires à l'application d'une politique linguistique (Calvet, 1987, p. 154-155).

En français on préfère aujourd'hui au terme de planification linguistique celui d'aménagement linguistique, qu'il est possible d'articuler globalement autour de deux pôles d'action. On peut d'une part aménager le statut des langues et leur corpus. Dans le premier cas, selon Heinz Kloss, l'intervention vise le statut social de la langue «par rapport à d'autres langues ou par rapport à un gouvernement national» (cité par Daoust, Maurais, 1997, p. 10). Dans le deuxième cas, $\mathrm{H}$. Kloss considère qu'il s'agit de l'aménagement de la langue elle-même, quand «un organisme, une personne ou des groupes de personnes visent un changement au niveau de la forme ou de la langue elle-même en proposant ou en imposant soit l'utilisation de nouveaux termes techniques, soit des changements au niveau de l'orthographe, soit encore un nouvel alphabet» (ouvrage cité, 1997, p. 9).

Nous nous intéressons à ce deuxième volet de l'aménagement linguistique qui concerne, entre autres domaines, la graphie de la langue, la création d'une écriture ou d'un système de transcription, le changement d'un type d'écriture à un autre, etc. qui font partie plus spécifiquement de l'élaboration linguistique, ou de l'élaboration du corpus. C'est précisément dans cette perspective que l'ex-Yougoslavie nous apparait comme un observatoire, un prisme à travers lequel on peut observer à quel point l'aménagement des langues est devenu une réalité du monde contemporain. Nous allons reprendre maintenant les trois phénomènes qui touchent à l'écriture à travers l'histoire, ceux de la cyrillisation et de la latinisation, largement présents dans cet espace, à différentes époques et à des degrés divers, et celui de l'arabisation, dont on ne peut parler qu'avec une certaine réserve dans la mesure où ce phénomène est limité à une époque très précise, d'une durée réduite, et sur un territoire restreint.

\section{La cyrillisation}

Le domaine de l'emploi de l'alphabet cyrillique peut paraitre de prime abord moins vaste que celui de l'alphabet latin, mais il recouvre un grand ensemble de langues, situé essentiellement au sud-est, à l'est de l'Europe et en 
Eurasie. Cet alphabet sert à noter les langues slaves de l'est, du sud et, de manière plus ou moins adéquate, un grand nombre de langues autres parlées dans l'ex-URSS, aussi diverses que des langues altaïques (langues turques d'Asie centrale, mongoles), finno-ougriennes (toutes, sauf les langues fenniques - finnois, estonien, carélien, live, vepse - et le hongrois), caucasiennes circassiennes (d'une complexité phonologique extrême) et dido-daghestaniennes, paléo-sibériennes.

L'alphabet cyrillique, récent ${ }^{2}$, établi par les disciples de Cyrille et Méthode pour adapter à la phonologie slave l'alphabet grec byzantin, a remplacé l'alphabet glagolitique. Le cyrillique fut ensuite, pour ceux qui ont subi les influences byzantines, l'alphabet qui représentait le lien avec la religion orthodoxe, et il est souvent mis en relation avec la «spiritualité» des peuples orthodoxes. Cet aspect-là a été largement exploité par les dirigeants nationalistes des pays de l'Est lors de la dernière décennie.

En ce qui concerne précisément l'espace yougoslave, alors que dans la Yougoslavie de Tito, l'égalité des deux alphabets avait été proclamée, la situation changea radicalement après l'éclatement du pays, non seulement dans le domaine juridique, mais aussi dans la pratique, en fonction des représentations identitaires et nationalistes. La Constitution de la RFY ${ }^{3}$ comporte de nombreux articles qui entendent règlementer la question linguistique. La langue officielle de la fédération est indiquée dans l'article 15, qui généralise l'usage du cyrillique et mentionne celui de l'alphabet latin:

En République fédérale de Yougoslavie, la langue serbe et ses parlers, l'ekavien et l'iékavien, ainsi que l'alphabet cyrillique sont officiels, tandis que l'alphabet latin est officiel selon les dispositions prévues par la Constitution et la loi $^{4}$ (Ustav Savezne Republike Jugoslavije, 1992).

En Serbie également, l'article 8 de la Constitution serbe fait du serbo-croate la langue officielle et du cyrillique l'alphabet officiel:

Le serbo-croate et l'alphabet cyrillique sont officiellement en usage dans la République de Serbie, tandis que l'alphabet latin est officiel conformément aux conditions fixées par la $1 \mathrm{i}^{5}$ (Ustav Republike Srbije, 1990).

2. Il a été créé au $10^{\mathrm{e}}$ siècle.

3. République fédérale de Yougoslavie, fondée à la suite de l'éclatement de l'ex-Yougoslavie, incluant Serbie et Monténégro.

4. "U Saveznoj Republici Jugoslaviji u sluzbenoj upotrebi je srpski jezik ekavskog i ijekavskog izgovora i cirilicko pismo, a latinicko pismo je u sluzbenoj upotrebi u skladu sa Ustavom i zakonom.»

5. «U Republici Srbiji u sluzbenoj je upotrebi srpskohrvatski jezik i cirilicno pismo, a latinicno pismo je u sluzbenoj upotrebi na nacin utvrdjen zakonom. » 
Dans la Yougoslavie actuelle, seule la République du Monténégro a proclamé l'égalité des deux alphabets dans sa Constitution. Autrement dit, deux dispositions ont remplacé dans la RFY le pluralisme scriptural qui précédait: régime à dominante cyrillique au niveau fédéral et en Serbie, régime pluraliste (latin et cyrillique) pour le Monténégro. Ce n'est pas seulement au niveau constitutionnel que le nationalisme scriptural est visible. En Serbie au début du régime de Milosevic, on tenta de «serbiser» la langue standard par le biais de l'alphabet: le régime ordonna l'impression des manuels scolaires exclusivement en cyrillique, excepté les manuels de langues étrangères, et les manuels en langues des minorités. En renonçant volontairement au bialphabétisme, on privait les enfants d'une formation qui ne pourrait être qu'un avantage pour leur avenir du point de vue de la diversité des compétences de communication écrite. Par ailleurs, certains périodiques imprimés jadis en alphabet latin passèrent l'un après l'autre au cyrillique. On insistait sur l'usage presque exclusif du cyrillique, en exigeant qu'il figure même dans la Constitution de la République de Serbie. Après une rectification qui devait modifier ce choix radical et la proposition que la Constitution «autorise» malgré tout l'alphabet latin dans certaines situations, ce projet fut abandonné. R. Bugarski souligne que cette politique ne pouvait aboutir, car «[elle] était appuyée par des émotions fortes, mais par des arguments faibles ${ }^{6}$ (Bugarski, 1997, p. 38).

Aujourd'hui, même après la chute du régime de Milosevic, le sentiment que l'alphabet cyrillique serait en danger continue d'être très présent dans certaines couches de la société serbe. Récemment a été formée une Association pour la protection du cyrillique ${ }^{7}$ qui sert davantage de forum pour des débats politiques nationalistes que de société savante, en travaillant à la promotion d'un élément culturel. À en croire certains observateurs, «leurs réunions servent à faire des tirades sur les complots mondiaux xénophobes ${ }^{8}$ (Gluhonjic, 2002), où on peut entendre que seuls «les mauvais Serbes écrivent en alphabet latin ${ }^{9}$ (Gluhonjic, 2002). Tout récemment, le 14 novembre 2002, 1'Assemblée nationale serbe a même voté une déclaration sur la protection du cyrillique, inquiétée par l'idée que cet alphabet reculerait de plus en plus devant l'alphabet latin, et «le manque de conscience du besoin de sauvegarder ses propres valeurs culturelles, l'identité nationale et ce qui la caractérise ${ }^{10}$ ( (Deklaracija o za?titi cirilice», 2002). Que le cyrillique recule devant les avancées de

6. « ... podrzan jakim emocijama ali slabim argumentima.»

7. Drustvo za zastitu cirilice.

8. «... koriste skupove za ksenofobicne tirade o belosvetskim zaverama. »

9. « ... losi Srbi pisu latinicom.»

10. «... odsustva svesti o potrebi negovanja sopstvenih kulturnih vrednosti, cuvanja nacionalnog identiteta i njegovih obelezja.» 
l'alphabet latin est effectivement un fait indéniable. Si l'on en croit les enseignants du secondaire, il arrive que les élèves leur demandent l'autorisation d'écrire leurs devoirs en alphabet latin, faute de connaitre suffisamment le cyrillique («Mnogi ne znaju cirilicu», 2002). De même, il est vrai que 18,5\% seulement des enseignes à Novi Sad sont en cyrillique et $31,8 \%$ à Belgrade ( $\mathrm{Ne}$ damo cirilicu», 2002). Mais de tels faits ne constituent pas en euxmêmes la menace d'une modalité d'écriture sur une autre et dépendent des infrastructures éducatives, de la diversité d'origine des populations urbaines dans les Balkans et d'échelles de véhicularité relative, jouant en faveur de l'alphabet latin. Autant de causes qui peuvent se traiter par un investissement éducatif et la tolérance pluraliste, plutôt que par la prescription forcée et le repli identitaire.

\section{La latinisation}

Après la christianisation effectuée par Rome, les Croates ont utilisé un certain temps le slavon croate et l'écriture glagolitique dans la liturgie avant de passer au latin. Dans l'ex-Yougoslavie, même si L'orthographe de la langue serbo-croate littéraire ${ }^{11}$ proclamait l'égalité des deux alphabets, il existait toujours une préférence en Croatie pour l'alphabet latin, comme d'ailleurs le cyrillique prévalait en Serbie. Pourtant, il est écrit dans ce livre de référence, publié en 1960, que «les deux alphabets, latin et cyrillique, sont égaux; pour cela il faut veiller à ce que les Serbes et les Croates apprennent au même titre les deux, ce qui peut se faire à travers le système éducatif» ${ }^{12}$ (Pravopis srpskohrvatskog knjizevnog jezika, 1960, p. 7). Après l'indépendance de la Croatie en 1991, les premières mesures du nouvel État sont prises: la République de Croatie ne s'appelle plus «socialiste», et la langue officielle est le croate, écrit en alphabet latin. La Constitution croate (art. 12) stipule que:

L'usage de la langue croate et l'alphabet latin sont officiels dans la République de

Croatie («La politique linguistique de croatisation», 2002).

Pourtant, l'usage officiel de l'alphabet cyrillique reste prévu dans les régions où les Serbes sont majoritaires. Ils peuvent continuer de nommer leur langue serbe, mais étant donné qu'ils représentent $12 \%$ de la population totale, ils sont traités comme les autres minorités linguistiques. On constate là encore le choix d'abandonner le pluralisme que représentait le bialphabétisme

11. Pravopis srpskohrvatskog knjizevnog jezika.

12. «Oba pisma, cirilica i latinica, ravnopravna su; zato treba nastojati da i Srbi $i$ Hrvati podjedanko nauce oba pisma, to ce se postici u prvom redu skolskom nastavom.» 
pour promouvoir le monoalphabétisme dans le cadre de la séparation, ce qui renforce le cloisonnement culturel dans l'ex-Yougoslavie.

\section{L'arabisation}

L'alphabet arabe a connu une très grande diffusion au Proche-Orient et au Maghreb avec l'expansion de l'islam. Il s'est imposé avant tout pour des raisons culturelles. Cette écriture transcrira également les langues de l'Empire ottoman jusqu'au début du $20^{\mathrm{e}}$ siècle. Sur le territoire de l'ex-Yougoslavie qui nous intéresse, l'islamisation de la population bosniaque du $15^{\mathrm{e}}$ siècle a été d'une très grande importance pour l'évolution de la langue littéraire en Bosnie. Le superstrat turc et l'arabe comme langue religieuse, ont laissé des traces dans la langue parlée par la population musulmane ${ }^{13}$ (Franolic, 1972, p. 27). En ce qui concerne l'alphabet, une forme particulière du cyrillique appelée bosancica, ou "le cyrillique serbe» a été en usage au Moyen Age en Bosnie (Garde, 1996, p. 130). La poésie bosniaque de langue slave a été écrite à l'époque ottomane en alphabet arabe aussi, appelé arabica et approprié au système phonologique serbo-croate, mais l'alphabet latin l'a rapidement remplacé (Dérens, Samary, 2000, p. 185).

Chaque État-nation proclamé après l'éclatement du pays commun veut se doter d'un emblème linguistique propre. La Bosnie-Herzégovine ne fait pas exception. Elle fut, comme d'ailleurs les autres républiques, une entité administrative officiellement multinationale où régnait un système complexe pour tenter de faire respecter les droits linguistiques et culturels de chacun. Dans le milieu musulman, on aspire aujourd'hui à souligner les éléments qui distinguent la langue bosnienne de la langue serbe. On garde pourtant l'alphabet latin et le parler iékavien, et cette variété se caractérise par beaucoup d'orientalismes, d'expressions locales, une abondance de termes d'origine turque ou arabe, surtout dans le domaine religieux. L'article 6 de la Constitution de la Fédération croato-musulmane de la République de Bosnie précise:

Les langues officielles de la Fédération sont la langue bosniaque [sic] et la langue croate. L'écriture officielle est l'alphabet latin («Les politiques linguistiques de la Bosnie», 2000).

En revanche, la langue officielle de la République serbe de Bosnie est le serbe et l'alphabet officiel est l'alphabet cyrillique. Il est intéressant de noter

13. Ceci est valable pour la population chrétienne également, puisque les Turcs sont restés longtemps dans la région. 
que, dans le cas de la composante musulmane du nouvel État de Bosnie-Herzégovine issu des guerres nationalistes récentes, un retour à l'arabisation n'a pas été envisagé, et que c'est l'option de l'alphabet latin qui a été retenue.

\section{Que montre le prisme yougoslave?}

À travers le prisme yougoslave, on peut saisir les enjeux politiques et identitaires de pays qui, à un moment ou un autre, ont choisi de se tourner vers un autre alphabet ou de modifier le leur, manifestant une intention de convergence culturelle entre tradition européenne occidentale (latinisation) et orientale (cyrillisation), parfois en dépit des conflits religieux et nationaux à petite échelle (latinisation plutôt qu'arabisation en Bosnie). Le choix des alphabets traduit des polarisations d'un ordre supérieur à celui de l'individuation des variétés linguistiques.

L'importance de l'alphabet latin est telle dans le monde occidental que l'on ne peut que comprendre ce qui a poussé Mustafa Kemal Atatürk à préférer l'alphabet latin à l'alphabet arabe, dans la volonté de tourner son pays vers l'Europe, de favoriser la communication internationale hors du seul monde musulman, et de marquer la fin d'un État théocratique en misant sur la laïcité. Cette réforme se justifiait aussi par des raisons structurales propres à la langue turque: l'alphabet arabe permettait difficilement de noter les voyelles de la langue turque et le système complexe et régulier de l'harmonie vocalique (palatale et labiale), mais il n'en reste pas moins que l'alphabet latin fut imposé en Turquie «moins parce qu'il transcrivait plus commodément les voyelles que parce qu'il permettait de bannir un grand nombre de mots du fond arabo-persan, traditionnellement notés en écriture arabe, et considérés par les Jeunes-Turcs comme les véhicules d'une idéologie archaïque, dont la modernisation du pays postulait l'abandon» (Hagège, 1994, p. 231). Les choix d'élaboration du corpus étaient donc multiples, et relevaient de l'intervention sur des facteurs inhérents (système vocalique et ses contraintes associatives) et contingents liés au contact de langues et de culture (rayonnement lexical arabo-persan).

D'une manière analogue en termes de politique de convergence culturelle, mais avec une finalité assimilationniste plus que de simple rayonnement culturel, la décision de généraliser l'écriture cyrillique en Union soviétique a fini par aboutir à une politique systématique de russification des langues et des ethnies, car «en luttant contre l'analphabétisme, on a favorisé la diffusion du credo communiste» (Février, 1984, p. 554). Il est caractéristique qu'en ces temps de «lendemains d'empire», le passage de l'alphabet cyrillique à l'alphabet latin soit déjà effectué pour certaines nouvelles nations de l'ex-URSS. 
Il peut également être envisagé pour d'autres, engageant ces pays vers de nouvelles configurations sociolinguistiques d'ordre majeur, à savoir, l'ordre impliqué par les aires culturelles caractérisées, entre autres facteurs historiques et sociolinguistiques, par les alphabets et les choix scripturaux.

C'est le cas de la langue azéri, appartenant au groupe turc, devenue langue officielle de l'Azerbaïdjan. Encore au $19^{\mathrm{e}}$ siècle, cette région de Transcaucasie penche davantage vers la latinisation que vers l'alphabet arabe, et la transition d'un système graphique à l'autre se fera à Bakou avant même de s'accomplir à Istanbul (Constant, 2002, p. 8-9). Cette langue changera plusieurs fois d'écriture au cours de l'histoire: l'alphabet latin remplace les caractères arabes «mal adaptés à la phonologie des langues turques» en 1924 (ouvrage cité, p. 26), puis la russification accompagnée d'un cyrillique aménagé à la phonétique de la langue commencera en 1940. Après l'indépendance, le pays réintroduira de nouveau l'alphabet latin en 1993. Il est évident que par ce geste le pays a choisi en même temps de tourner le dos à la Russie et à l'Iran et de se rapprocher de la Turquie. Lors de la célébration de cet évènement, le président Gueïdar Aliev a déclaré au sujet de l'alphabet latin, «celui-ci est maintenant bel et bien le nôtre» (CDCA, 2001). On ne peut éviter de se demander pour combien de temps encore.

C'est également le cas de l'ouzbek, autre langue turque qui a connu à peu près le même sort que l'azéri: passage relativement rapide de l'alphabet arabe à l'alphabet latin (de 1927 à 1940), puis au cyrillique (dès 1938), puis de nouveau à l'alphabet latin, après l'indépendance du pays. Si le cyrillique a été introduit pour juguler le panturquisme et rapprocher les nations turques d'Asie centrale du "grand frère» slave (Uhres, 1996), aujourd'hui l'intention est «surtout de s'en éloigner» (Uhres, 1996), et de tirer autant que possible un trait sur cette forme de colonialisme que fut le régime soviétique, et sur ses corrélats, comme la russification sous ses diverses formes. De même, le turkmène, langue officielle du Turkménistan, écrite en caractères arabes jusqu'en 1920, puis à partir de 1926, dans une variante d'alphabet latin proche de celle appliquée au turc anatolien de la Turquie kémaliste, puis en cyrillique depuis 1939 et les réformes staliniennes, se tourne de nouveau, en 1996, vers l'alphabet latin. Encore une fois, et ceci nous parait également vrai pour les pays voisins, «cette décision de changement d'alphabet traduit davantage une volonté politique affichée, plutôt qu'une décision linguistique qui exprimerait avec précision les réalités phoniques et phonologiques du turkmène moderne et standard» (Blacher, 1996).

Un autre exemple est celui du tadjik, langue indo-iranienne d'Asie centrale qui appartient à l'ensemble des nouvelles langues nationales issues de l'effondrement de l'Union soviétique. Alors que le choix de l'alphabet latin dans 
d'autres pays d'Asie centrale ex-soviétique contribue à couper les racines religieuses dans les régions où l'islam est encore assez puissant et à faciliter la convergence culturelle avec l'espace turc anatolien, le choix tadjik de revenir à l'alphabet arabo-persan est tout autre. C'est certainement pour le Tadjikistan autant une manière de se défendre de l'influence du russe, que le désir de s'affirmer dans le monde islamique, voire de converger avec le reste du monde indo-iranien au-delà des divisions ethniques imposées par le découpage du pays lors de sa fondation dans le cadre soviétique (Roy, 1997, p. 115). Après une longue période de soviétisation, les Tadjiks ont de nouveau renoué avec leur tradition en découvrant un héritage littéraire et religieux rendu inaccessible pendant plusieurs décennies. Cette redécouverte du patrimoine peut aussi s'expliquer par le fait que «l'islam militant a connu une montée en puissance indéniable» ces derniers temps (Jeannot-Jahangiri, 2001, p. 104), particulièrement pour un pays dont l'indépendance récente s'est accomplie dans un contexte de guerre civile.

Un dernier exemple pour illustrer le lien relatif entre langue, choix de modalité écrite et processus d'assimilation politique et culturelle, tiré de l'histoire européenne cette fois, sera celui du lituanien. Par rapport aux cas que nous venons d'examiner, dans le cadre soviétique récent, il est moins connu que le lituanien a été soumis à une politique de cyrillisation au $19^{\mathrm{e}}$ siècle, précisément en 1840 quand «l'utilisation de l'alphabet latin pour écrire la langue lituanienne fut interdite» et remplacée par l'utilisation de l'alphabet cyrillique (Dumoulin, Vermès, 1997). Ce n'est qu'en 1904 que les «autorités russes permirent l'édition d'ouvrages lituaniens en caractères latins» (ibid.). La position de la Lituanie dans ce grand ensemble que fut l'ex-URSS, et la longue tradition de la langue lituanienne ont rendu la perspective d'une nouvelle cyrillisation impossible. Autant d'exemples qui montrent que le phénomène n'est pas propre aux Balkans et que les reconfigurations géostratégiques et politiques sont souvent accompagnées de reconfigurations sociolinguistiques qui incluent les systèmes d'écritures.

Ce bref voyage à travers le temps, des Balkans à l'Asie centrale, montre que d'une part il a toujours été important pour tous de rattacher la langue à un alphabet particulier, et qu'il existe un lien entre l'expansion des écritures et le rayonnement culturel et politique d'autre part: celui des religions, mais aussi celui de la laïcité dans le cas du turc anatolien. Le cas de la communauté linguistique serbo-croate est, de ce point de vue des multiples modalités du rayonnement culturel et des choix de convergence, très révélateur. Nous avons surtout évoqué des exemples relevant des Balkans d'une part et des pays du 
Caucase et de l'Asie centrale d'autre part, tant les deux régions se ressemblent sur le plan géostratégique. Le Caucase, «les Balkans de l'Eurasie» selon les mots de Z. Brzezinski (Thual, 2001, p. 13), est également un carrefour de civilisations, un espace où le monde chrétien et le monde musulman se croisent et se rencontrent. Pour ce qui est de l'ex-Yougoslavie, la question que l'on se pose forcément est la suivante: s'arrêtera-t-on là? $\mathrm{Si}$ «la désintégration une fois commencée impose une autre logique, différente ${ }^{14}$ (Bugarski, 2001, p. 40), on peut effectivement supposer que la séparation politique de la communauté serbo-croate pourrait, à long terme, éloigner encore davantage ces langues si proches. Le choix de l'alphabet peut se révéler de ce point de vue très important, puisque les systèmes d'écritures apparaissent, tout comme la langue, comme des éléments relevant des attributs sociaux de l'identité. Un alphabet peut se transformer en symbole de l'identité d'un peuple et mobiliser à son tour en lui des sentiments collectifs, être l'objet d'un attachement, porteur d'«implications culturelles provoquant des sentiments d'identification aussi forts que celles de la langue elle-même» (Siguan, 1996, p. 115). Mais, il est très important de comprendre, nous semble-t-il, que ni le cyrillique ni l'alphabet latin ne peuvent être considérés comme des "alphabets nationaux» spécifiques ou essentiellement inhérents aux peuples qui se les arrachent aujourd'hui, dans le but de s'éloigner encore plus lorsque la langue est réinvestie comme un lieu de conflit.

\section{Bibliographie}

BLACHER Ph., 1996, «La langue turkmène et son écriture à l'aube du $21^{\mathrm{e}}$ siècle», Bulletin de l'OAC, nº 1, février 1996.

BUGARSKI R., 1997, Jezik od mira do rata [La langue de la paix à la guerre], Belgrade, $\mathrm{XX}$ vek.

BUGARSKI R., 2001, Lica jezika [Les visages de la langue], Belgrade, XX vek.

CALVET L.-J., 1993, L'Europe et ses langues, Paris, Plon.

CALVET L.-J., 1999, La guerre des langues et les politiques linguistiques, Paris, Hachette, (première édition 1987.

CDCA, http://www.cdca.asso.fr/gamk/2001.

CONSTANT A., 2002, L'Azerbaïdjan, Paris, Éditions Karthala.

DAOUST D., MAURAIS J., 1997, «L'aménagement linguistique», dans Maurais J. (dir.), 1997, Politique et aménagement linguistiques, Québec, Les publications du Québec, p. 7-46.

«Deklaracija o zastiti cirilice» [Déclaration sur la protection du cyrillique], 2002, Belgrade, Sluzbeni list SRJ, n 61/2002.

14. «... jednom zapoceta dezintegracija namece novu, drukciju logiku.» 
DÉRENS J.-A., SAMARY C., 2000, Les conflits yougoslaves de A à Z, Paris, Les Éditions de l'Atelier.

DUMOULIN B., VERMÈS G., 1997, «La pratique des langues dans une situation pluriculturelle comme révélateur des sentiments identitaires: le cas des enfants lituaniens, russes et polonais de Vilnius en $1996 »$, DiversCité Langues, en ligne, vol. II, disponible sur le site www.uquebec.ca/diverscite

FÉVRIER J., 1984, Histoire de l'écriture, Paris, Payot (première édition 1959).

FRANOLIC B., 1972, La langue littéraire croate: aperçu historique, Paris, Nouvelles éditions latines.

GARDE P., 1996, «Langue et nation: le cas serbe, croate et bosniaque », dans SERIOT P. (éd.), 1996, «Langue et nation en Europe centrale et orientale du $18^{\mathrm{e}}$ siècle à nos jours», Cahiers de l'ILSL, n ${ }^{\circ}$, p. 123-148.

GLUHONJIC D., 2002, «Ko je smestio cirilici?» [Qui a travaillé contre le cyrillique?], http://www.panonijapress.co.yu/046/cirilica046.htm

HAGĖGE C. 1994, Le souffle de la langue, Paris, Odile Jacob.

JEANNOT-JAHANGIRI G., 2001, «L'instabilité régionale en Asie centrale», Weber O., Afghanistan : la mémoire assassinée, UNESCO/Mille et une nuits, p. 95107.

«La politique linguistique de croatisation», site http: // www.tlfq.ulaval.ca/axe/europe / croatie.htm

«Les politiques linguistiques de la Bosnie», site http:// www.tlfq.ulaval.ca/axe/europe/bosnieherzacc.htm

«Mnogi ne znaju cirilicu» [Nombreux sont ceux qui ne connaissent pas le cyrillique], 2002, Politika, 20 juin 2002.

«Ne damo cirilicu» [Nous défendons le cyrillique], 2002, Danas, Belgrade, 8 nov. 2002.

Pravopis srpskohrvatskog knjizevnog jezika [L'orthographe de la langue serbo-croate littéraire], 1960, Novi Sad — Zagreb, Matica srpska - Matica hrvatska.

ROY O., 1997, La nouvelle Asie centrale, ou la fabrication des nations, Paris, Seuil.

SIGUAN M., 1996, L'Europe des langues, Sprimont, Mardaga.

THOMAS P.-L., 1998, «La dislocation linguistique comme instrument de pouvoir: l'exemple de l'ex-Yougoslavie», dans CHAKER S. (éd.), Langues et pouvoir de l'Afrique du Nord à l'Extrême Orient, Aix-en-Provence, Édisud, p. 109-121.

THUAL F., 2001, Le Caucase, Paris, Flammarion.

UHRES J.1996, «Introduction de l'alphabet ouzbek à graphie latine», Bulletin de l'OAC, $\mathrm{n}^{\circ} 1$, février 1996.

Ustav Republike Srbije [Constitution de la République de Serbie], 1990, Belgrade, Sluzbeni list.

Ustav Savezne Republike Jugoslavije [Constitution de la RFY], 1992, Belgrade, Sluzbeni list. 